\title{
Электрически активные состояния захвата и переноса заряда, обусловливающие медленную рекомбинацию в кристаллах бромида таллия при низких температурах
}

\author{
(C) В. Кажукаускас, Р. Гарбачаускас, С. Савицки
}

Кафредра ффизики полупроводников и Институт прикладных исследований, Вильнюсский университет, LT-10257 Вильнюс, Литва

E-mail: vaidotas.kazukauskas@ff.vu.It

(Получена 28 июня 2017 г. Принята к печати 5 июля 2017 г.)

\begin{abstract}
Исследовались образцы монокристаллов $\mathrm{TlBr}$, выращенных по методу Бриджмена-Стокбаргера. Установлено, что при межзонном возбуждении светом при температурах ниже $200 \mathrm{~K}$ проявляются эффекты замороженной проводимости. При этом выявляются ярко выраженные сверхлинейные зависимости индуцированной (фото-) проводимости от напряженности приложенного электрического поля. Как свидетельствуют результаты исследования термостимулированной проводимости, эти явления могут быть связаны с заполнением ловушечных состояний с энергиями термической активации $0.08-0.12$ эВ. Это состояние может быть снято в результате термического гашения при температурах $\gtrsim 180 \mathrm{~K}$ из-за опустошения заполненных после оптической генерации энергетических состояний с энергией активации $0.63-0.65$ эВ.
\end{abstract}

DOI: $10.21883 / F T P .2018 .02 .45439 .8672$

\section{1. Введение}

Бромид таллия (TlBr) характеризуется электрофизическими параметрами, которые делают его чрезвычайно привлекательным для создания детекторов ионизирующего рентгеновского и $\gamma$-излучения, способных действовать при комнатной температуре. Этот полупроводниковый материал имеет большую ширину запрещенной зоны, 2.68 эВ, высокую плотность, 7.56 г/ $\mathrm{cm}^{3}$, и большие атомные номера: $\mathrm{Tl}-81, \mathrm{Br}-35$ [1-3]. Большая ширина запрещенной зоны обеспечивает низкую скорость тепловой генерации носителей заряда, поэтому материал обладает высоким удельным сопротивлением, превышающим $10^{10} \mathrm{Oм} \cdot$ см при комнатной температуре, а приборы на его основе - низкими темновыми токами и малыми шумами. Высокая плотность и большие атомные номера обеспечивают высокую эффективность остановки квантов. Пиксельные детекторы $\gamma$-излучения на основе $\mathrm{TlBr}$ имеют энергетическое разрешение для $\gamma$-квантов с энергиями на полуширине 122 и 662 кэВ соответственно 6.7 кэВ (5.5\%) и 22.3 кэВ (3.4\%) [2,3]. Важный параметр для чувствительности прибора произведение подвижности электронов и их времени жизни - достигает $\mu \tau=4.1 \cdot 10^{-3} \mathrm{~cm}^{2} / \mathrm{B}$ при комнатной температуре [4]. Тем не менее, как было показано недавно, свойства и электрические параметры детекторов на основе $\mathrm{TlBr}$ чувствительно зависят от особенностей технологических процессов, что приводит к различным плотностям дефектов [5].

Более того, одной из главных проблем, которая по существу по-прежнему ограничивает практическую применимость $\mathrm{TlBr}$, является наличие ионной проводимости, сильно зависящей от температуры [6], что вызывает неустойчивость характеристик устройств [7]. Это приводит, например, к ухудшению спектроскопических характеристик из-за поляризационных эффектов, если устройства работают при комнатной температуре [2]. Электромиграция примесей в $\mathrm{TlBr}$ была, например, исследована в [8]. Кроме того, ионный перенос и возникающие в результате реакции могут вызвать появление дефектов Шоттки [9]. Образование пар вакансий Т1 и $\mathrm{Br}$ в объеме происходит в результате создания новых состояний на поверхности кристалла, занятых $\mathrm{Tl}$ и Br. К тому же границы зерен и дислокации могут также служить источниками и(или) стоками дефектов в результате их образования или аннигиляции [9]. В [10] оценены энтальпии образования дефектов Шоттки $(0.91$ эВ), миграции катионов $(0.51$ эВ) и миграции анионов $(0.28$ эВ).

Кроме того, относительно мало исследованы перенос носителей заряда и свойства электрически активных дефектов $\mathrm{TlBr}$ в зависимости от условий возбуждения.

$\mathrm{B} \mathrm{TlBr}$, как и во всех полупроводниковых материалах с высоким удельным сопротивлением, явления генерации и переноса носителей существенно изменяются с температурой и при возбуждении светом, что позволяет выявлять эффекты, связанные с уровнями дефектов и неоднородностями кристаллов [11-13]. Поэтому нашей задачей было исследование (фото-) проводимости $\mathrm{TlBr}$ в области температур ниже комнатной до температуры жидкого азота. Так как при температурах ниже $240 \mathrm{~K}$ ионная проводимость $\mathrm{TlBr}$ становится пренебрежимо малой [13], такие условия являются наиболее подходящими для анализа электронного переноса заряда.

\section{2. Образцы и методика эксперимента}

Монокристаллы T1Br были выращены методом Бриджмена-Стокбаргера. Для получения образцов с размера- 
ми $\sim 4 \times 2 \times 1$ мм монокристаллы разрезались, после чего образцы полировались механически и химически. На концах образцов напылялись золотые электроды. Мы исследовали спектры фотопроводимости, термически стимулированные токи и переходные характеристики тока после возбуждения в температурном диапазоне от 300 до $100 \mathrm{~K}$ в зависимости от приложенного напряжения смещения. Подача напряжения питания и измерения тока, протекающего через образец, осуществлялись прибором Keithley 6430B Source-Measurement Unit.

Для исследования спектральных зависимостей фототока (I) образцы возбуждались монохроматическим светом с помощью монохроматора Leitz. Для выделения уровней дефектов спектры анализировались с использованием модели фотоионизации глубоких уровней Луковского [14]

$$
I \propto n_{M} \Delta E_{M}^{0.5}\left(h v-\Delta E_{M}\right)^{1.5} /(h v)^{3} .
$$

Здесь $\Delta E_{M}$ - энергия оптической активации глубокой ловушки, $n_{M}$ - концентрация заполненных уроней, $h v$ - энергия квантов возбуждающего света.

Перенос и захват носителей исследовали с помощью спектроскопии термически стимулированых токов (ТCT), методика применения которой подробно описана в [15]. Образцы охлаждались в криостате жидкого азота в темноте, после этого возбуждались белым светом для заполнения электрически активных дефектных состояний. После выключения света измерялась кинетика долговременных релаксаций тока. ТСТ измерялись при постоянной скорости нагрева, составляющей $10 \mathrm{~K} / \mathrm{Mин}$. Для анализа возможных эффектов, вызванных тепловой генерацией носителей одновременно из нескольких близко расположенных центров захвата, была использована информативная методика многократного нагрева, позволяющая последовательно опустошать близлежащие уровни, обеспечивая тем самым их разрешение [15]. При таком же напряжении на образцах измерялись также темновые токи. Они были вычтены из данных по ТСТ, полученных после возбуждения светом. ТСТ, вызванные термической генерацией носителей заряда из ловушек, можно описать как [16]

$$
I=\frac{1}{2} e L A f_{t} n_{t 0} \exp \left[-\frac{E_{t}}{k T}-\frac{f_{t} k T^{2}}{\beta\left(E_{t}+k T\right)} \exp \left(-\frac{E_{t}}{k T}\right)\right],
$$

где $n_{t 0}$ - плотность захваченных носителей на ловушках с энергией активации $E_{t}, T-$ температура, $\beta-$ скорость нагрева, $k$ - постоянная Больцмана, $f_{t}$ - частота попытки высвобождения носителей, $e$ - элементарный заряд, $L-$ длина образца, $A-$ площадь его сечения.

Изначальное заполнение ловушек $n_{t 0}$ оценивалось путем интегрирования экспериментальных кривых по времени. Полученные значения показали хорошее совпадение с параметрами численного моделирования кривых ТCT.

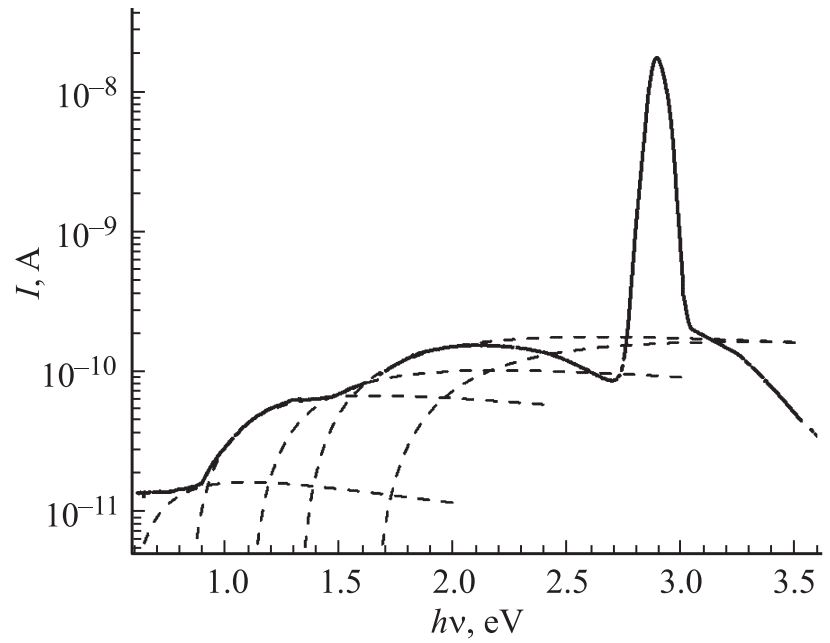

Рис. 1. Спектр фототока (сплошная линия) и его численная аппроксимация (штриховые кривые) при $240 \mathrm{~K}$.

\section{3. Результаты и их обсуждение}

Обычно при комнатной температуре фотопроводимость в $\mathrm{TlBr}$ мала по сравнению с темновой проводимостью, поэтому фотоспектроскопия неприменима для исследования дефектов [17]. Однако темновой ток уменьшается экспоненциально с уменьшением температуры, а энергия тепловой активации этого процесса $\sim(0.78-0.8)$ эВ [13]. Поэтому при низких температурах эффект фотопроводимости заметно возрастает, что позволяет идентифицировать уровни дефектов в зависимости от предыстории образца, т.е. его возбуждения светом и(или) напряжением [13].

На рис. 1 представлены экспериментальные спектральные зависимости при $240 \mathrm{~K}$ и их аппроксимация по формуле (1). При $\sim 2.75$ эВ проявляется яркий максимум, вызванный межзонной генерацией носителей. Падение тока на стороне более высоких энергий от этого максимума связано с увеличением поглощения света и, следовательно, усилением влияния поверхностной рекомбинации. При аппроксимации экспериментальных спектров были получены следующие оценки энергии фотоионизации: $0.55,0.83,1.1,1.32$ и 1.65 эВ. Перезарядка ловушки с энергией активации 1.65 эВ может индуцировать изменения подвижности носителей, как это наблюдалось в работе [13].

Характерно, что дефектные состояния в области между 1 и 2.5 эВ проявлялись по-разному в зависимости от предварительного возбуждения образца. „Плечи“ в спектрах фотопроводимости с энергиями $\sim 0.83$ и $\sim 1.1$ эВ, связанные с оптическим возбужденим дефектов, появлялись, если спектры сканировали начиная от высоких энергий квантов. Влияние уровней с энергиями 1.32 и 1.65 эВ уменьшалось, если образцы выдерживались под напряжением в течение нескольких часов [13]. 


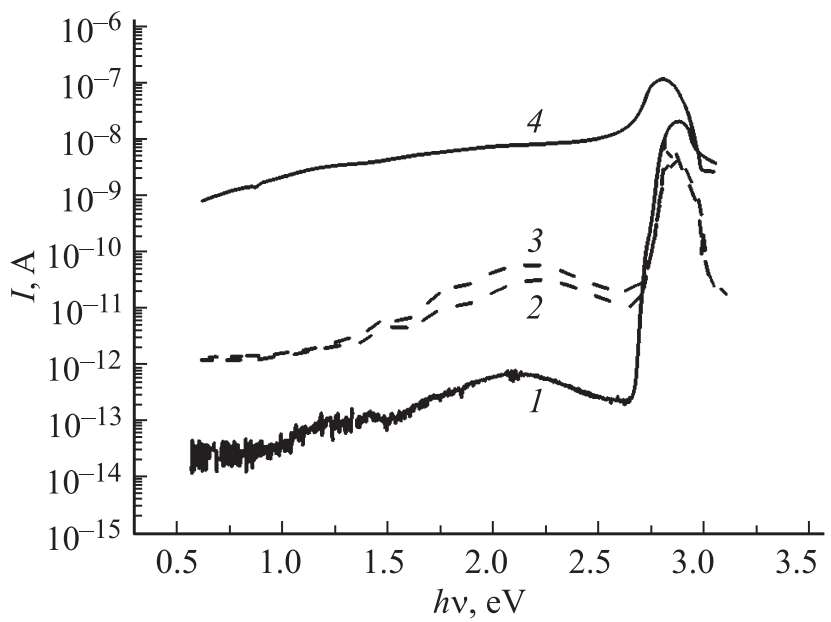

Pис. 2. Спектры фототока при разных температурах: 115 (сплошные кривые) и $240 \mathrm{~K}$ (штриховые). Сначала измерения проводились при увеличении энергии квантов возбуждающего света $(1,2)$, после этого при уменьшении $(3,4)$.

Аналогичная сложная структура дефектов была обнаружена и в спектах фотолюминесценции (ФЛ) $[18,19]$. В работе [18] линии ФЛ между $1.5-2.0$ эВ наблюдались при рентгеновском возбуждении. Это было объяснено влиянием диффузионно-контролируемой рекомбинации. Вместе в тем в работе [19] линии ФЛ при 1.1 эВ наблюдались в кратковременных спектрах поглощения при возбуждении импульсным электронным пучком, и объяснялось это захватом дырок катионными вакансиями $\left(\mathrm{Tl}^{2+} V^{c-}\right)$. Такой центр может создаваться при захвате дырок вакансиями, созданными при предварительном облучении или же в результате образования пары Френкеля $\left(\mathrm{Tl}^{2+} V^{c-}\right.$ и межузельный атом таллия $\left.\mathrm{Tl}_{i}^{0}\right)$. Из наших результатов следует, что такая вакансия может появиться также в результате возбуждения светом, даже если интенсивность света, выходящего из монохроматора, мала. Более подробный анализ результатов, полученных при $240 \mathrm{~K}$, представлен в [13].

На рис. 2 представлены спектральные зависимости фотопроводимости того же образца при разных температуpax: 240 и $115 \mathrm{~K}$. Чтобы предотвратить предвозбуждение образцов световыми квантами с высокими энергиями, спектры сначала измерялись начиная с низких энергий. После этого сканирование проводилось в обратном направлении. Видно, что при увеличении энергий квантов спектры, измеренные при обеих температурах, выглядят одинаково, если принять во внимание уменьшение темнового тока при низкой температуре. Характерно, что спектры, измеренные в обоих направлениях при $240 \mathrm{~K}$ (и ниже до $\sim 190 \mathrm{~K}$ ), почти совпадают (рис. 2), что свидетельствует об отсутствии каких-либо длительных процессов.

Однако при более низких температурах $(\lesssim 180 \mathrm{~K})$ после межзонного возбуждения образцов их фоточувствительность остается примерно на 4 порядка выше, чем до возбуждения. Более того, характерная дефектная структура в спектрах уже не проявляется. Это связано с проявлением индуцированной замороженной фотопроводимости, для которой характерны длительные релаксации [20]. Такие долговременные релаксации тока после межзонного возбуждения образцов светом, которые могут быть объяснены уменьшением рекомбинации, наблюдались и в нашем случае. Характерно, что даже после нескольких часов релаксации ток не падал до нуля, а насыщался на постоянном уровне, превышающем темновой ток.

Эффект замороженной фотопроводимости связан с уменьшением рекомбинации генерированных светом носителей заряда, поскольку они попадают в энергетические состояния захвата, разделенные в пространстве. Такие состояния захвата могут быть обусловлены потенциальными неоднородностями краев запрещенной зоны. Поэтому захваченные носители локализируются в пространстве, и для того чтобы перемещаться в пространстве и рекомбинировать им необходима дополнительная энергия для высвобождения из ловушек [20]. В работе [20] указано, что ток обычно релаксирует, следуя одной экспоненциальной зависимости. Однако в нашем случае для аппроксимации результатов одной, даже растянутой, экспоненты было недостаточно:

$$
I_{S C}=I_{S C}(t=0) \exp \left[\left(-\frac{t}{\tau}\right)^{\beta}\right], t_{1 / 2}=\tau(\ln 2)^{1 / \beta},
$$

где $I_{S C}-$ ток короткого замыкания, $\tau$ и $\beta$ - характерные параметры растянутой экспоненциальной зависимости, а $t_{1 / 2}-$ время уменьшения тока в 2 раза. В нашем случае для аппроксимации экспериментальных зависимостей релаксаций потребовалась сумма трех экспонент, что указывает на весьма комплексный эффект. Характерные константы времени этих трех экспоненциальных составляющих были соответственно: $140-160,480-540$ и $1800-2100 \mathrm{c}$.

Медленные релаксации тока и подвижности носителей заряда наблюдались и в других полупроводниках с высоким удельным сопротивлением, как, например, в полуизолирующем GaAs [21-23]. В этом состоянии проявляются ярко выраженные сверхлинейные зависимости тока от приложенного электрического поля. Из рис. 3 видно, что при увеличении энергии квантов, преобладает линейная зависимость тока от приложенного напряжения (линейные вольт-амперные характеристики), однако при измерении спектров начиная с высоких энергий различия в фототоке достигают нескольких порядков величины - вольт-амперные характеристики становятся сверхлинейными.

Для того чтобы исследовать эти ловушечные состояния с низкой вероятностью рекомбинации при низких температурах, мы провели измерения термически стимулированных токов. На рис. 4 пунктирной кривой представлена экспериментальная ТСТ, измеренная при напряженности электрического поля 200 В/см. При 


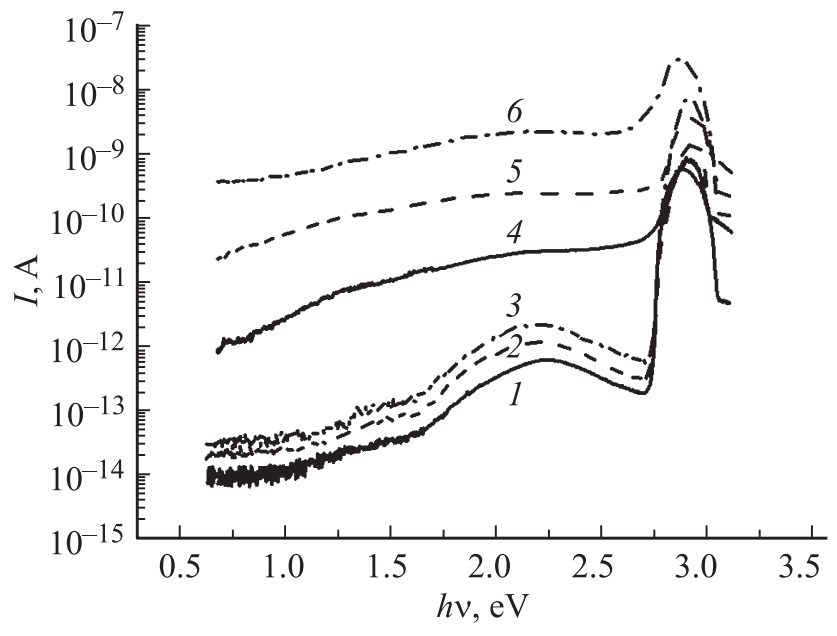

Рис. 3. Спектры фототока при $115 \mathrm{~K}$ и разных напряженностях электрического поля: сплошная линия -200 B/см, штриховая $-600 \mathrm{~B} / \mathrm{cm}$, штрихпунктирная $-1000 \mathrm{~B} / \mathrm{cm}$. Сначала измерения проводились при увеличении энергии квантов возбуждающего света $(1-3)$, после этого при уменьшении (4-6).

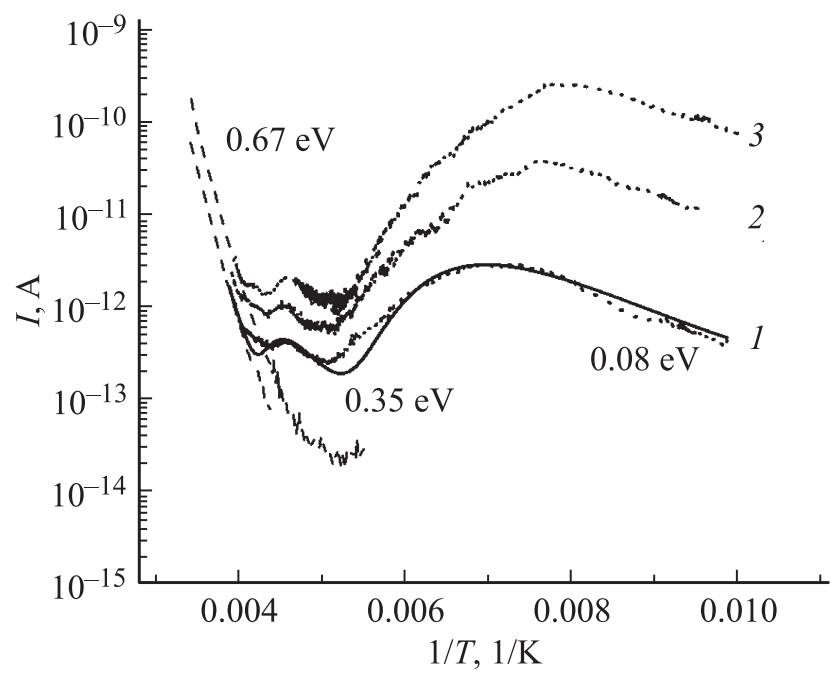

Рис. 4. ТСТ, измеренные при разных напряженностях электрического поля, В/см,: $1-200,2-600,3-1000$. Пунктирные кривые - экспериментальные данные ТСТ, сплошная кривая - численная аппроксимация данных при $200 \mathrm{~B} / \mathrm{cm}$, штриховые кривые - темновой ток.

низких температурах ярко видны два максимума ТСТ с различной энергией активации. При приближении к комнатной температуре кривые ТСТ опять начинают совпадать с темновым током, имеющим энергию активации $\sim 0.65$ эВ. До температур $\sim(180-200) \mathrm{K}$ при увеличении напряжения выявляется яркая нелинейная зависимость тока от напряжения. При более высоких температурах зависимость тока от приложенного напряжения снова становится линейной.

Для более детального анализа такого поведения были измерены ТСТ в режиме многократного нагрева, резуль- таты которых показаны на рис. 5. При низких температурах на всех трех кривых наблюдается один ярко выраженный широкий и высокий максимум ТСТ, имеющий энергию термической активации (0.065-0.08) эВ. В следующих циклах нагрева эти значения увеличиваются до $0.11-0.12$ эВ. Это можно объяснить тем, что при термической генерации захваченных носителей из потенциальных ям рельефа краев запрещенной зоны эффективная глубина частично опустошенных потенциальных ям увеличивается при каждом следующем температурном цикле, так как уменьшается количество оставшихся захваченных носителей и они заполняют лишь все более глубокие состояния в потенциальных ямах. После полного опустошения этих ловушек начинается генерация носителей из более глубоких состояний

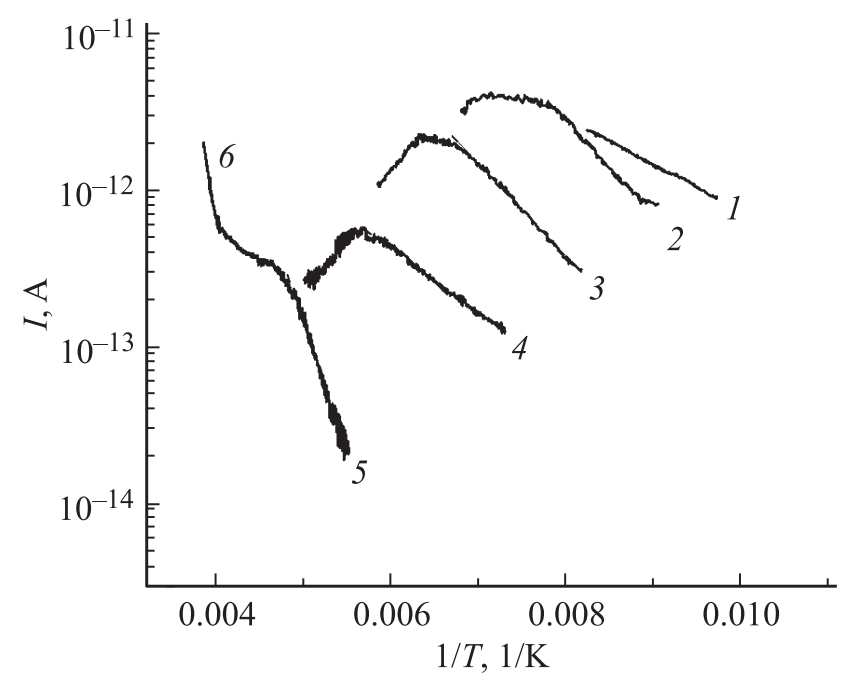

Рис. 5. ТСТ, полученные методом многократного нагрева. Энергии тепловой активации соответствующих участков, эВ: $1-0.07,2-0.11,3-0.11,4-0.08,5-0.33,6-0.66$.

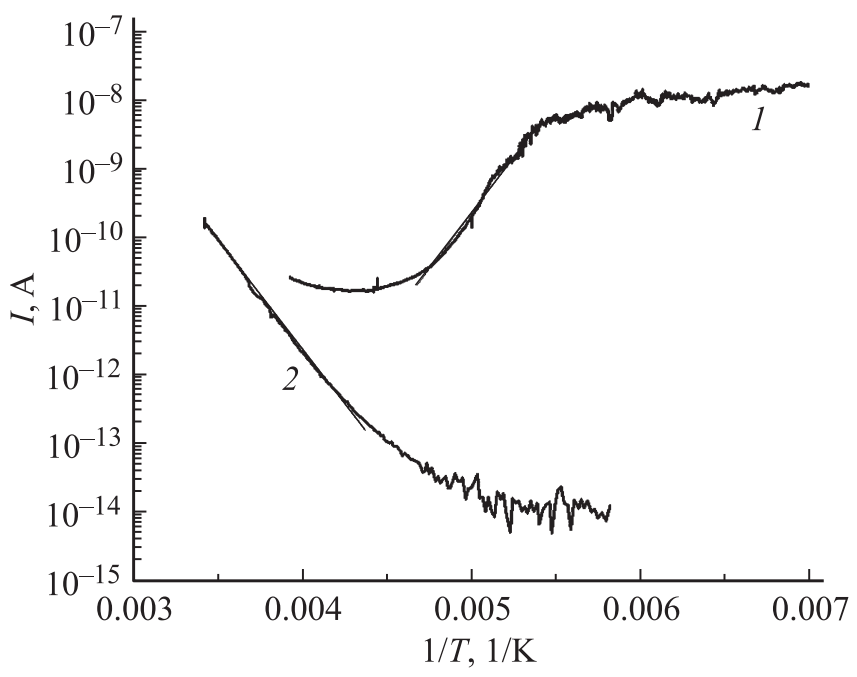

Рис. 6. Температурные зависимости фототока при межзонном возбуждении (1) и темнового тока (2), измеренные от низких температур. 
с энергией термической активации $\sim 0.33$ эВ. Как видно по характеру кривых ТСТ, опустошение этого уровня происходит при $\sim 220 \mathrm{~K}$. После этого начинается тепловая генерация с энергией активации 0.65 эВ, которая близка к значениям 0.68-0.7 эВ, приведенным в работах $[17,24]$. Мы использовали эти экспериментальные значения в качестве параметров модели для аппроксимации кривой ТСТ согласно формуле (2), что показано на рис. 4 сплошной кривой.

Наличие дефектных состояний, которые в зависимости от их заполнения могут начать действовать как центры рекомбинации, подтверждается эффектом термического гашения фототока в освещенных образцах. На рис. 6 показаны температурные зависимости темнового тока и фототока при межзонном возбуждении светом. Они измерялись начиная с низких температур. Фототок сильно превышает ТСТ, и он остается почти стабильным до $\sim 180 \mathrm{~K}$. При повышении температуры ток начинает падать с энергией активации $\sim 0.65$ эВ. Такое тепловое гашение фотопроводимости хорошо известно, в частности в полупроводниках $\mathrm{A}^{\mathrm{II}} \mathrm{B}^{\mathrm{VI}}[25]$. Это происходит в результате термического опустошения рекомбинационных центров, которые в заполненном состоянии не могут захватывать носители и обеспечивать их рекомбинацию. Характерно, что энергии активации как темнового тока, так и процесса теплового гашения совпадают. Это доказывает, что тепловое гашение может происходить в результате опустошения ловушки с энергией активации $\sim 0.65$ эВ.

\section{4. Заключение}

С целью исследования электронных процессов захвата и переноса заряда в $\mathrm{TlBr}$, обусловленных дефектными состояниями, избегая влияния ионного переноса, проявляющегося при высоких температурах, мы провели исследования фотоэлектрических спектров и термически стимулированных токов, в которых основное внимание уделялось эффектам при температурах ниже комнатной и до $100 \mathrm{~K}$. Впервые в $\mathrm{TlBr}$ идентифицированы особенности фото-термо-электрических явлений, связанные с проявлением замороженной индуцированной (фото-) проводимости, проявляющиеся при температурах ниже $200 \mathrm{~K}$ и вызванные межзонным возбуждением светом. Для этого состояния характерно уменьшение вероятности рекомбинации, приводящее к долговременной релаксации (фото-) проводимости. Характерно, что при этом наблюдаются ярко выраженные сверхлинейные вольтамперные характеристики. Указанное состояние связано с заполнением ловушечных состояний с энергиями термической активации 0.08-0.12 эВ, вероятно, обусловленных потенциальным рельефом краев запрещенной зоны. Состояние замороженной индуцированной (фото-) проводимости может быть снято в результате термического гашения при температурах $\gtrsim 180 \mathrm{~K}$ из-за опустошения заполненных в результате оптической генерации энерге- тических состояний с энергией активации $0.63-0.65$ эВ. Кроме того, в исследованных кристаллах выявлено несколько фотоактивных дефектов с энергиями оптической активации $0.55,0.83,1.1,1.32$ и 1.65 эВ, а также электрически активный уровень ловушек при 0.35 эВ.

\section{Список литературы}

[1] P.J. Sellin. Nucl. Instrum. Meth. A, 513, 332 (2003).

[2] K. Hitomi, M. Matsumoto, O. Muroi, T. Shoji, Y. Hiratate. IEEE Trans. Nucl. Sci., 49, 2526 (2002).

[3] K. Hitomi, M. Matsumoto, O. Muroi, T. Shoji, Y. Hiratate. J. Cryst. Growth, 225, 129 (2001).

[4] K. Hitomi, T. Onodera, T. Shoji, Y. Hiratate, Z. He. IEEE Trans. Nucl. Sci., 55, 1781 (2008).

[5] R.A. dos Santos, C.H. de Mesquita, J.B.R. da Silva, C. de Melo Ferraz, F.E. da Costa, J.F.T. Martins, R.F. Gennari, M.M. Hamada. Adv. Mater. Sci. Eng., 2017, 1750517 (2017).

[6] T. Onodera, K. Hitomi, T. Shoji. Nucl. Instrum. Meth. A, 568, 433 (2006).

[7] K. Hitomi, T. Shoji, K. Ishii. J. Cryst. Growth, 379, 93 (2013).

[8] K.H. Kim, E. Kim, H. Kim, R. Tappero, A.E. Bolotnikov, G.S. Camarda, A. Hossain, L. Cirignano, R.B. James. J. Appl. Phys., 114, 133701 (2013).

[9] S.R. Bishop, H.L. Tuller, M. Kuhn, G. Ciampi, W. Higgins, K.S. Shah. Phys. Chem. Chem. Phys., 15, 11926 (2013).

[10] S.R. Bishop, W. Higgins, G. Ciampi, A. Churilov, K.S. Shah, H.L. Tuller. J. Electrochem. Soc., 158, J47 (2011).

[11] J. Vaitkus, V. Kažukauskas, R. Kiliulis, J. Storasta. Inst. Phys. Conf. Ser., 136, 755 (1994).

[12] R. Kiliulis, V. Kažukauskas, J. Storasta, J.-V. Vaitkus. J. de Physique I, 6, 1165 (1996).

[13] V. Kažukauskas, A. Ziminskij, J.V. Vaitkus, V. Gostilo, M. Shorohov. Nucl. Instrum. Meth. A, 607, 123 (2009).

[14] G. Lucovsky. Solid State Commun., 3, 299 (1965).

[15] G. Kavaliauskienė, V. Kažukauskas, V. Rinkevičius, J. Storasta, J.V. Vaitkus, R. Bates, V. O’Shea, K.M. Smith. Appl. Phys. A. Mater. Sci. Proc., 69, 415 (1999).

[16] J.G. Simmons, G.W. Taylor. Phys. Rev. B, 5, 1619 (1972).

[17] V. Kažukauskas, A. Jurgilaitis, J.-V. Vaitkus. Proc. SPIE, 6596, C5960 (2007).

[18] L. Grigorjeva, D. Millers. Nucl. Instrum. Meth. B, 191, 131 (2002).

[19] L. Grigorjeva, D. Millers. Electrochem. Soc. Proc., 98, 438 (1998).

[20] М.К. Шейнкман, А.Я. Шик. ФТП, 10 (2), 209 (1976). M.K. Sheinkman, A.Y. Shik. Sov. Phys. Semicond., 10, 128 (1976).

[21] V. Kažukauskas. J. Appl. Phys., 84, 2053 (1998).

[22] V. Kažukauskas, J. Vaitkus. Z. Phys. B. Condens. Matter, 94, 401 (1994).

[23] J. Vaitkus, R. Baubinas, E. Gaubas, K. Jarašiūnas, R. Jašinskaite, V. Kažukauskas, E. Kuprusevičius, J. Matukas, V. Palenskis, J. Storasta, M. Sūdžius, R. Tomašiūnas. Nucl. Instrum. Meth. A, 466, 39 (2001).

[24] J. Vaitkus, J. Banys, V. Gostilo, S. Zatoloka, A. Mekys, J. Storasta, A. Žindulis. Nucl. Instrum. Meth. A, 546, 188 (2005).

[25] R.H. Bube. Photoconductivity of Solids (N.Y., John Wiley and Sons, Inc., 1960).

Редактор Л.В. Шаронова 


\section{Electrically active states of charge capture and transport causing slow recombination in thallium bromide crystals at low temperatures}

V. Kažukauskas, R. Garbačauskas, S. Savicki

Semiconductor Physics Department and Institute of Applied Research, Vilnius University, LT-10257 Vilnius, Lithuania

Abstract In order to investigate the electron capture and transport processes due to defect states in $\mathrm{TlBr}$, the photoelectric spectra and thermally stimulated currents were studied, focusing on effects at temperatures below room temperature and down to $100 \mathrm{~K}$. For the first time in $\mathrm{TlBr}$ the features of photo-thermoelectrical effects related with the phenomena of the frozen induced (photo-) conductivity were identified at temperatures below $200 \mathrm{~K}$, which could be induced by interband excitation by light. This state is associated with the filling of the trapping states with thermal activation energies of $0.08-0.12 \mathrm{eV}$. The state of the frozen induced (photo-) conductivity can be removed as a result of thermal quenching above about $180 \mathrm{~K}$, because of the emptying of the energy states, initially filled due to optical generation, with an activation energy of $0.63-0.65 \mathrm{eV}$. 